\title{
An Automated Test System for Measuring and Improving Electrosurgical Effects
}

\author{
Anup Amin ${ }^{1,2}$, Daniel Friedrichs ${ }^{2}$, Carolyn Ford ${ }^{2}$, Eric Larson ${ }^{2}$, and James Gilbert ${ }^{2}$ \\ ${ }^{1}$ University of Texas at Arlington, Arlington, TX, USA \\ ${ }^{2}$ Covidien Surgical Solutions, Boulder, CO, USA
}

\begin{abstract}
Designing instruments and power supplies for electrosurgery (a critical tool in modern surgical practice) is a difficult proposition; a large number of competing variables affect an even larger number of electrosurgical performance metrics. This paper describes the design of an apparatus used to tightly control experimental variables, record high-speed data from numerous sensors, and process the results into a form which better elucidates the underlying physical mechanisms and gives insight into potential design improvements.
\end{abstract}

Index Terms -Electrosurgery, automated testing, data acquisition

\section{INTRODUCTION}

Electrosurgery is a technology regularly employed in modern operating rooms to enable tissue division and dissection with concomitant hemostasis. When passed through tissue, high-frequency electrical current can cause sufficient joule heating to cause both a cutting and coagulating effect [1]. Electrosurgery is an indispensable tool in modern surgical practice, finding use in over $80 \%$ of procedures performed in the United States [2].

Despite the importance and prevalence of electrosurgery, the technology has seen only superficial changes since its conception by Dr. William T. Bovie in 1920 [3]. Further, the physical mechanisms underlying the electrosurgical effect are still not fully elucidated, partially owing to the large number of involved variables which influence a large number of competing metrics of surgical effectiveness. For instance, high peak voltages from the electrosurgical instrument tend to improve hemostasis, at the expense of producing more smoke which obscures vision and is potentially mutagenic or carcinogenic [4]. In general, performance metrics of interest to the surgeon include cutting speed, level of hemostasis, cutting force, smoke/steam produced, electric arcs produced, and consistency [6]. The variables that influence these metrics include tissue type, tissue volume, applied voltages and currents, cutting speed, and instrument geometry. The inability to experimentally control this large set of interconnected variables has previously obstructed efforts to better understand the underlying physical mechanisms of electrosurgery.
The goal of this work is to construct an apparatus which will allow consistent and repeatable control over several contributory variables, in order to better isolate parameters which may improve performance of electrosurgical tools. Section II of this paper describes the design and construction of said apparatus, detailing methods of improving control and facilitating isolated measurements. Section III presents several example results garnered from the apparatus, illustrating the benefits of precise and repeatable control. Section IV summarizes the results and offers future research directions.

\section{THE GANTRY SYSTEM}

The test apparatus (termed the "Gantry") is described in terms of its hardware components (Section IIA), and software components (Section IIB).

\section{A. Hardware}

The hardware for the experimental apparatus consists of a system which acquires and processes signals, and a system which automates a test procedure to yield consistent results by eliminating human-user-induced variability. The hardware can thus be broken into two categories: (1) sensors, and (2) motion control.

\section{1) Sensors}

The sensors connected to the experimental apparatus allow measurement of the following physical phenomena:

a) Voltage and Current: These electrosurgical signals are used to calculate power and impedance. Accurately measuring voltage is difficult because of the high-frequency of the AC current $(500 \mathrm{kHz})$, the high potential difference generated (up to $10 \mathrm{kV}$ ), and the fact that the electrosurgical generator output is not ground-referenced. A Tektronix ${ }^{\circledR}$ P5205A $100 \mathrm{MHz}$ high-voltage differential voltage probe overcomes these obstacles and permits wide-bandwidth measurement of the voltage. The current measurement also needs to be wide-bandwidth, as well as both DC and AC coupled. A Tektronix ${ }^{\circledR}$ TCP0030 AC/DC current probe satisfies these requirements.

b) Force: The drag force on the surgical instrument is another metric that affects the user's perceived quality of the electrosurgical cut. The drag force varies as the type of tissue varies, but is also dependent on power supply parameters. Torque on the electrosurgical instrument is measured by a high-sensitivity flange-mounted $5 \mathrm{oz} /$ in reaction torque 
sensor pivoted at one end of the electrosurgical blade. Amplification of the force sensor signal is accomplished using a National Instruments PXI-4220 sensor input card.

c) Arcing: The intensity of arcs produced results in hemostasis around the cut area, and the quality of hemostasis depends on the consistency of power dissipation around the area of incision. Parameters affecting the quality of hemostasis include power, depth of incision and the nature of tissue. Quantifying the arcing phenomenon through the duration of the cut assists in characterizing the aforementioned issues. The intensity of arcing between the electrosurgical blade and tissue is quantified using a Thorlabs APD110A2 photodetector coupled to an optical fiber and lens assembly which selectively passes only UV light (effectively isolating arc luminescence from background light and any infrared energy emitted from the hot cutting blade).

d) Depth of cut: Another factor which has a sizeable effect on the drag force is the depth at which the incision is made. It is important to determine the amount of drag force met at a particular depth into the tissue, as this also contributes to the user's perception of effectiveness. The depth is measured using an Optex CD33-120NV laser profiler, aimed parallel to the electrosurgical blade.

e) Smoke detection: Electrosurgical smoke is an undesirable product of electrical cauterization. Apart from blocking vision during laparoscopic procedures it poses a potential health hazard. A moderately high-speed video camera is used in a preliminary attempt to quantify smoke production using a spatial algorithm and thresholding.

Figure 1 shows a block diagram of the experimental setup, illustrating the voltage, current, depth, arc, smoke, and torque sensors, and detailing their integration with the dataprocessing system.

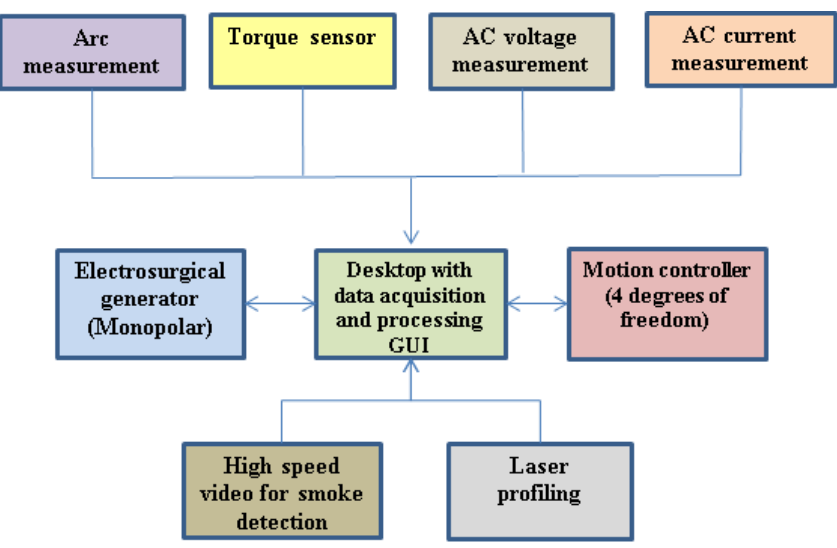

Fig. 1. Block diagram of the automated electrosurgical testing apparatus.

\section{2) Motion Controller (“Gantry”)}

The complete system is assembled into a gantry-like structure with a high performance motion controller which has four degrees of freedom: $\mathrm{X}$-axis, $\mathrm{Y}$-axis, Z-axis and rotational (at the pivotal end of the electrosurgical instrument). The tissue sample is placed on a mantle which has two degrees of freedom along the $\mathrm{Y}$-axis and $\mathrm{Z}$-axis. With freedom along the $\mathrm{Y}$-axis, the mantle adjusts the distance between the tissue and the tip of the instrument. The electrosurgical instrument is mounted on a second motion controller which has two degrees of freedom (along the Yaxis and rotational in directions parallel to the plane of $\mathrm{Y}$ axis). The horizontal distance between the tissue and the tip of the electrosurgical blade is traversed with the help of this controller. All four controllers offer control over both velocity and acceleration.

Fig. 2 illustrates the complete Gantry system.

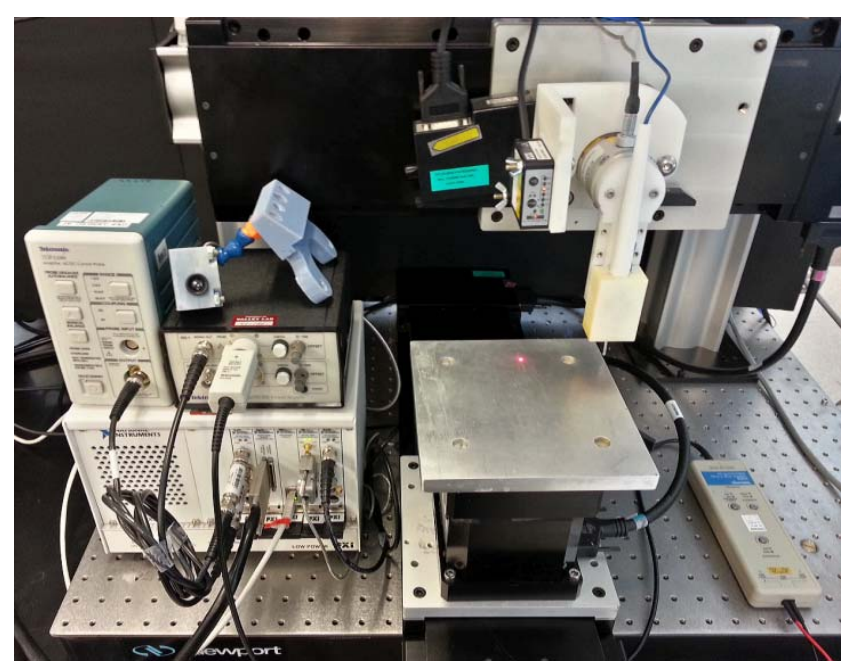

Fig. 2. Experimental "Gantry" setup with component sensors and motion controller.

\section{B. Software}

The supporting software is an application developed in National Instruments LabVIEW ${ }^{\text {TM }}$ software with the requisite motion controller drivers. The software performs the tasks of establishing control of the motion controller, acquiring data, and processing the collected data.

\section{1) Motion Control}

The software provides two options for motion control: (1) The user can manually manipulate the motion of the controller in each of the four degrees of freedom; (2) The user can initiate a 'Batch-Cut'. This option allows the user to pre-orchestrate the cutting process. The user can configure the number of cuts desired, the distance between cuts, the power setting of each cut, and the cutting speed.

\section{2) Data Acquisition and Processing}

Sensor data is collected by a high-speed National Instruments PXI-5922 digitizer card with a sampling speed of $10 \mathrm{Ms} / \mathrm{s}$. Force readings are made by a National 
Instruments $^{\mathrm{TM}}$ PXI-4220 signal conditioner, with a maximum of $200 \mathrm{kS} / \mathrm{s}$ sampling speed.

Collected data is processed in several ways. The raw current and voltage sampled from the system are processed mathematically to obtain the impedance and power over each AC cycle. The torque sensor data establishes a relation of force versus power and force versus cutting speed. The arc intensity measurement is compared to sampled voltage and current to correlate arc events with electrical phenomena.

To time-efficiently calculate the tissue impedance (a parameter used to estimate tissue hydration and temperature, among other things) the Goertzel algorithm is used to perform a discrete Fourier transform at the supply frequency. This simplifies and accelerates the processing of voltage and current data given a fixed frequency supply. A typical Goertzel filter to compute the power spectrum for voltage and current at a given supply frequency, $\omega$, is given by (1) and (2) respectively.

$$
\begin{gathered}
V(n)=v(n)+2 \cos (2 \pi \omega) V(n-1)-V(n-2) \\
I(n)=v(n)+2 \cos (2 \pi \omega) I(n-1)-I(n-2)
\end{gathered}
$$

\section{RESULTS}

This section presents a number of preliminary results collected using the Gantry system which includes analysis of parameters like Power and Impedance (Section III A), Force and Power (Section III B), Force and Cutting Depth (Section III C) and a brief analysis of important parameters such as Arcing (Section III D), and Smoke (Section III E).

\section{A. Power and Impedance}

This sub-section compares results of power and impedance measurements for cuts made by the gantry to that made by a human user.

Figure 3 shows the resulting cycle-by-cycle average power delivered and calculated tissue impedance, collected during a test activation of the experimental apparatus.

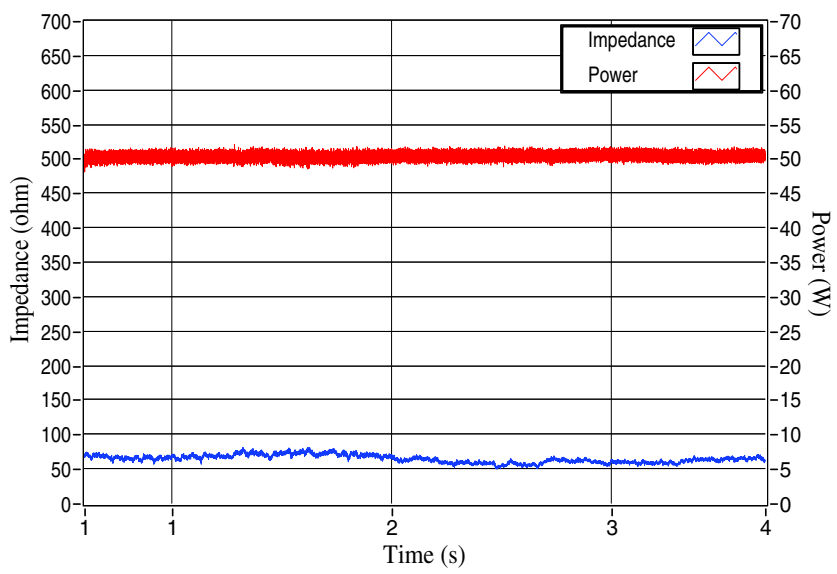

Fig. 3. Power and impedance using Gantry
In Fig. 3, relatively constant power and impedance are plotted, which is consistent with the expected result of cutting through a uniformly thick and homogeneous tissue sample at a constant speed and power setting. Figure 4 shows similar power and impedance plots generated when a human user attempted to maintain a constant speed and depth of cut.

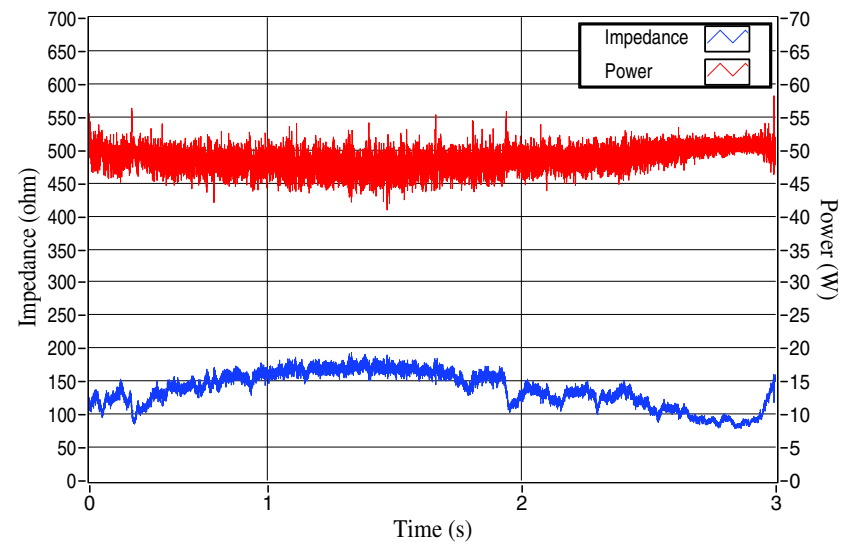

Fig. 4. Power and impedance using human operator.

Clearly, the Gantry system confers the advantage of consistency. The ability to deliver constant power, independent of user-induced variation in cut speed or depth, will allow exploration of tissue heating effects, predictions of tissue temperature rise, and additional measurements and computations.

\section{B. Force vs. Power}

Figure 5 illustrates how changing the delivered power affects drag force. Note that the time-dependent increase in force is a result of a non-uniformly-flat tissue sample, yet the result still clearly demonstrates a reduction in drag force as applied power is increased.

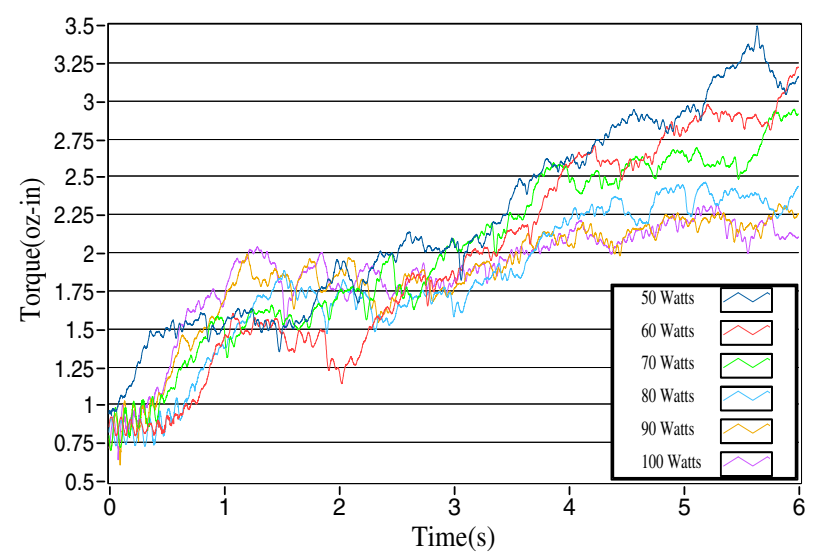

Fig. 5. Drag force versus applied power. 


\section{Force vs. Depth}

Figure 6 shows drag force measurements for various cutting depths when the instrument was set to a constant velocity and a constant power.

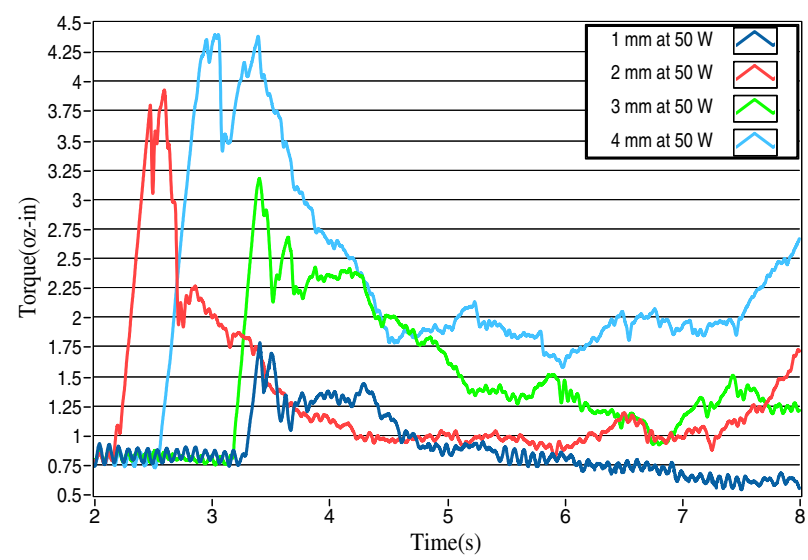

Fig. 6. Drag force versus cutting depth.

As anticipated, the drag force is directly proportional to the depth at which the incisions are made. By establishing better control over the output power, the drag force at the point of incision can be maintained at a constant value ensuring uniform and consistent cuts at any depth.

\section{Arc Detection}

Optical intensity data collected by the photodetector is sampled at the same rate as raw voltage and current facilitating the correlation of the arc events with electrical output. Figure 7 graphs the effect variable power setting has on the average measured optical intensity.

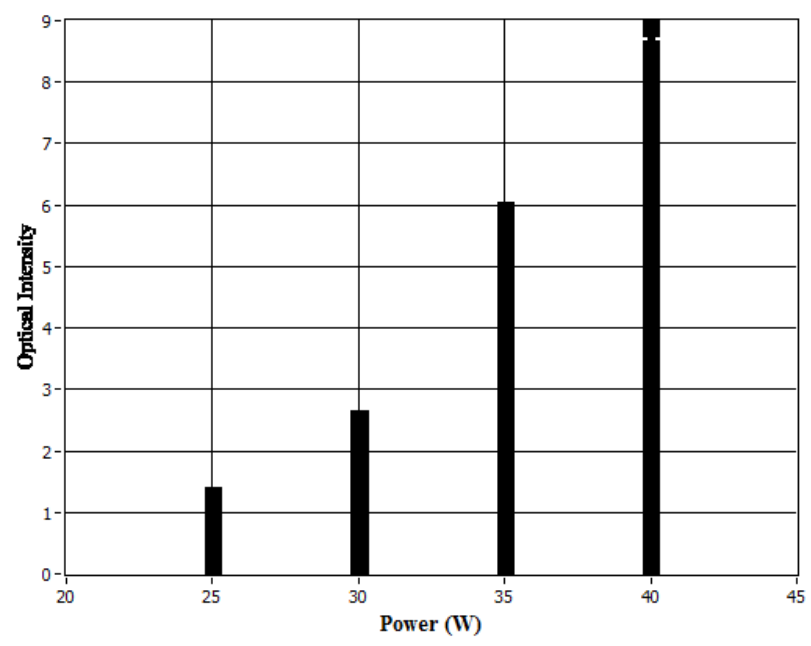

Figure 7. Optical intensity versus Power.

It can be inferred from the above graph that as the power increases there is a noticeably linear ramp in the intensity of arcs.

\section{E. Smoke Detection}

Spatial algorithm and thresholding is applied to frames acquired from high speed video recordings of the electrocauterization process to extract smoke traces invisible to the naked eye, providing a measure of the rate of smoke generation at different points along the length of the incision.

Thresholding identifies other fixed objects which object detection tends to eliminate, but a large portion of the smoke can be seen here, which was not readily apparent in the original image. Figure 8 shows the effects of thresholding on a frame, highlighting smoke traces normally invisible in the regular frame.

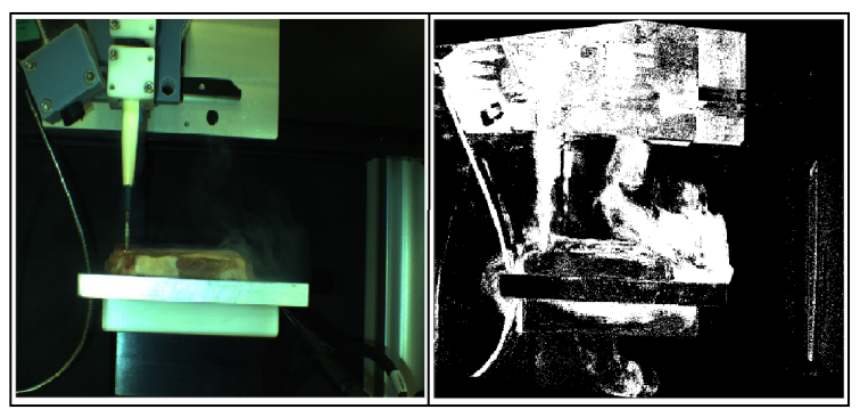

Fig. 8. Image enhanced after 'Thresholding' highlighting smoke.

It is desirable to know the convection of the surgical smoke under a normal surgical environment as well as monitor its volume in order to moderate its obtrusive effects.

\section{CONCLUSIONS}

This paper presents a unique electro-mechanical setup that is employed to acquire information about various electrosurgical phenomena. The use of an experimental apparatus to precisely control speed- and depth-of-cut, while simultaneously recording multiple high-resolution data streams, promises to add significant value to the process of designing electrosurgical instruments and power sources.

\section{ACKNOWLEDGEMENTS}

Authors wish to acknowledge significant contributions by Sara Anderson, Gary Couture and Kenlyn Bonn in design and construction of much of the motion control apparatus, and by Robert Lathrop for initial LabVIEW ${ }^{\mathrm{TM}}$ coding to support the motion control and data acquisition.

\section{REFERENCES}

[1] N. N. Massarweh, N. Cosgriff, D. P. Slakey, "Electrosurgery: History, Principles, and Current and Future Uses," Journal of the American College of Surgeons, vol. 202, no. 3, pp. 520530, March 2006.

[2] Friedrichs, D.A.; Erickson, R.W.; Gilbert, J.; , "A New Dual Current-Mode Controller Improves Power Regulation in 
Electrosurgical Generators," Biomedical Circuits and Systems, IEEE Transactions on , vol.6, no.1, pp.39-44, Feb. 2012

[3] J. A. Pearce, Electrosurgery. New York: Wiley, 1986.

[4] Helmut, W; Jurgen, L; Hans Jurgen, W; Rainer, M; Hans Dieter, L; "Characterization of tissue interaction by analysis of electrosurgical smoke" Engineering in Medicine and Biology Society, 1995. IEEE 17th Annual Conference.

[5] W. M. Honig, "The Mechanism of Cutting in Electrosurgery," IEEE Transactions on Biomedical Engineering, pp. 58-62, Jan. 1975.

[6] L. S. Feldman, P. R. Fuchshuber, D. B. Jones, Eds., The SAGES Manual on the Fundamental Use of Surgical Energy (FUSE). New York, NY: Springer Science+Business Media, 2012. 